\title{
Relação entre a resistência ao cisalhamento e a resistência à compressão paralela às fibras de madeiras folhosas
}

\author{
Relationship between shear strength and compressive \\ strength parallel to grains of hardwoods
}

\author{
Natalia Garcia Couto \\ João Paulo Boff Almeid a \\ José Silvio Govone \\ André Luis Christoforo \\ Francisco Antonio Rocco Lahr
}

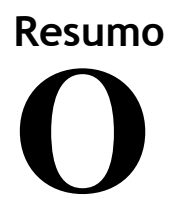

documento normativo NBR 7190:1997 estabelece os métodos de ensaio para obtenção das propriedades físicas e mecânicas do material, porém as premissas exigem procedimentos realizados em laboratórios especializados e de alto custo. Considerando a impossibilidade da realização dos ensaios, o próprio documento estabelece relações entre propriedades visando facilitar a caracterização do material. No que se refere às relações entre as propriedades de resistência ao cisalhamento e a resistência à compressão paralela às fibras a norma apresenta valores distintos em seu texto. $\mathrm{O}$ trabalho objetivou investigar, considerando os resultados de ensaios experimentais, a relação entre essas propriedades obtidas com auxílio do método dos mínimos quadrados, considerando para tanto dez espécies de madeiras do grupo das folhosas. Os resultados foram comparados com os valores de relações estabelecidas pela NBR 7190:1997, em que o coeficiente ótimo encontrado considerando o conjunto de todas as espécies foi $86,4 \%$ superior ao apresentado pela norma brasileira. Em relação à variação da relação entre as propriedades investigadas considerando as diferentes classes de resistência da madeira no presente estudo, não foi identificado decréscimo nos valores dos coeficientes ótimos conforme as classes aumentam (situação que a NBR 7190:1997 expõe em

${ }^{1}$ Natalia Garcia Couto 1 Universidade Federal de São Carlos São Carlos - SP - Brasil

${ }^{2}$ João Paulo Boff Almeida ${ }^{2}$ Universidade Federal de São Carlos São Carlos - SP - Brasil

${ }^{3}$ José Silvio Govone ${ }^{3}$ Universidade Estadual Paulista Juliode
Mesquita Filho Rio Claro - SP - Brasil

${ }^{4}$ André Luis Christoforo ${ }^{4}$ Universidade Federal de São Carlos São Carlos - SP - Brasil

${ }^{5}$ Francisco Antonio Rocco Lahr

${ }^{5}$ Universidade de São Paulo São Carlos - SP - Brasil

Recebido em 15/01/19 Aceito em 01/04/20 seu texto), apresentando assim outro resultado divergente.

Palavras-chave: Compressão paralela às fibras. Cisalhamento paralelo às fibras. Mínimos quadrados. Relações entre propriedades.

\section{Abstract}

In Brazil, the standard NBR 7190: 1997 establishes testing methods to determine the physical and mechanical properties of the material. However, the tests require that the procedures be undertaken in specialised, high-cost laboratories. The Brazilian code sets different values for relations between shear strength and compression strength parallel to the grain. The aim of this study was to investigate the relation between compressive strength and shear strength parallel to the grain, considering the results of the tests of those properties. The results were compared with the relation values setby the Brazilian Standard NBR 7190:1997, and the optimal coefficient found was $86.4 \%$ higher than the coefficient set by the Brazilian standard, considering the set of all species. With regards to the variation in the relation between the investigated properties, considering the different classes of wood resistance in this study, no decreases were found in the values of the optimal coefficients as the classes increase (a situation described in the NBR 7190:1997 standard), which represent one further divergent result.

Keywords: Compressive strength parallel to the grain. Shear strength parallelto the grain. Least squares. Relation between properties. 


\section{Introdução}

A madeira é um material muito utilizado desde os primórdios da humanidade, esteve presente em atividades humanas habituais, como a superação de obstáculos naturais, transporte, agricultura, entre outros. Por ser um material versátil, foi sempre fundamental para as necessidades humanas, e a sua ampla disponibilidade foi um fator importante para uma grande expansão na sua utilização na construção de edificações, pontes, silos e telhados (CHRISTOFORO et al., 2013).

A alta resistência em relação a sua massa é uma característica importante e de destaque da madeira, sendo essa relação três vezes maior que para o aço e dez vezes maior que para o concreto. Já em relação à competitividade a madeira tem melhor desempenho em relação a outros materiais do ponto de vista econômico em base de custos iniciais, além de apresentar vantagens quando comparado seu custo em longo prazo (CALIL JÚNIOR; DIAS, 2005).

Remage et al. (2017) destacam a eficiência da madeira em estrutura longa ou extensa, nas quais uma parte significativa da carga a ser suportada é seu próprio peso. Além disso, em relação às forças sísmicas, como as ações impostas à estrutura depende fortemente de sua massa, estruturas mais pesadas tendem a sofrer forças sísmicas maiores. Por isso, as edificações com elementos estruturais em madeira apresentam bom desempenho nesse tipo de solicitação.

A madeira é um material biodegradável, reutilizável e reciclável. Edifícios de aço e concreto incorporam e consomem de $12 \%$ a $20 \%$ mais energia do que edifícios de madeira, considerando a energia utilizada na obtenção do material como no consumo ao longo do ciclo de vida da edificação. Construir com madeira também resulta na redução de resíduos, prédios de aço e concreto produzem de $6 \%$ a $16 \%$ mais resíduos sólidos do que a madeira, tanto na fabricação quanto na construção (WANG; TOPPINEN; JUSLIN, 2014).

O Brasil possui cerca de 8.715 espécies de árvores em seu território, o que corresponde a $14 \%$ das 60.065 que existem no planeta, segundo o estudo realizado por Beech et al. (2017). Em relação à cobertura florestal, o Brasil possui uma área significativa, seja de nativas ou plantadas. No que diz respeito às nativas, segundo o Sistema Florestal Brasileiro (2016) a área é de aproximadamente 485,8 milhões de hectares, o que corresponde a $58 \%$ do território total do país. Em relação às florestas plantadas, em 2016 a área era de aproximadamente 7,84 milhões de hectares, segundo a Indústria Brasileira de Árvores (2017).

A utilização de madeira como material de construção para habitações residenciais está bem estabelecida em vários países da Europa, no Reino Unido, por exemplo, aproximadamente 20\% das novas residências são em estruturas de madeira, na Escócia esse percentual é cerca de 70\% (REMAGE et al., 2017). Já de acordo com Hemstrom (2016), no Japão 45\% das construções residenciais são em wood frame, na Nova Zelândia esse percentual é de $85 \%$, chegando aos impressionantes $90 \%$ na América do Norte.

Apesar de possuir um grande potencial madeireiro, o Brasil não tem explorado devidamente esse material na construção civil. Dentre os fatores que limitam tal emprego, destacam-se as restrições de natureza históricocultural, o desconhecimento das espécies e de suas propriedades, a escassez de mão de obra qualificada e de investimentos no setor (CURY; TOMAZELLO FILHO, 2011).

Outro preconceito referente à construção de edificações com sistemas estruturais em madeira é em relação à combustibilidade do material e à insegurança diante de potenciais riscos relacionados a incêndios. Essa situação é fruto do desconhecimento do comportamento da madeira em relação ao fogo (MORAES; FIGUEROA, 2009). Calil Júnior e Dias (2005) afirmam que grandes peças de madeira têm boa resistência ao fogo, com desempenho superior a outros materiais em condições severas de exposição. Os autores explicam que isso ocorre porque em uma situação de incêndio forma-se uma camada de carvão externamente nas peças de madeira, e como o material não possui boa condutividade térmica, essa camada se torna isolante e evita que a temperatura interna nas peças aumente demasiadamente, mantendo parte de sua resistência, diferente do que ocorre, por exemplo, em peças metálicas, onde a seção se aquece rapidamente devido a sua alta condutividade.

Laroca e Matos (2003) destacam que a evidente vocação florestal brasileira poderia auxiliar no controle do déficit habitacional, que constitui um problema social de enormes proporções no país. Para isso, os autores evidenciam a necessidade da difusão de informações referente aos benefícios das construções em madeira e das características do material, principalmente para o mercado consumidor. Entre as informações que devem ser conhecidas e divulgadas estão as propriedades da madeira (físicas, químicas, mecânicas e anatômicas). Almeida et al. (2014) destacam que o conhecimento dessas propriedades é imprescindível para uma utilização racional do material e um correto dimensionamento.

320 Couto, N. G.; Almeida, J. P. B.; Govone, J. S.; Christoforo, A. L.; Lahr, F. A. R. 
No Brasil, a Associação Brasileira de Normas Técnicas (ABNT), por meio do seu documento normativo número 7190 de 1997 (ABNT, 1997), intitulado "Projeto de Estruturas de Madeira", estabelece as premissas e os métodos de cálculo para dimensionamento das estruturas em madeira, assim como os métodos de ensaio para obtenção das propriedades físicas e mecânicas do material. A norma exige procedimentos realizados em laboratórios especializados e com equipamentos de alto custo para a obtenção das propriedades das espécies de madeira. Considerando a impossibilidade da obtenção dessas propriedades via experimentação, a norma brasileira propõe algumas relações entre propriedades com base no ensaio de compressão paralela às fibras.

Para as folhosas, que são usualmente utilizadas em projetos estruturais devido a sua alta resistência, a NBR 7190 (ABNT, 1997) estabelece, no item 6.3.3, entre outros, que a resistência característica ao cisalhamento paralelo às fibras $(\mathrm{fv} 0, \mathrm{k})$ é de $0,12(12 \%)$ da resistência característica à compressão paralela às fibras $(\mathrm{fc} 0, \mathrm{k})$, sem fazer distinção de classes de resistências. Entretanto, na Tabela 9 (Classes de resistências das dicotiledôneas), página 16, a norma apresenta valores de resistência característica à compressão e ao cisalhamento paralelo às fibras divididos em classes, sendo a relação entre essas propriedades variáveis de $0,20(20 \%)$ a $0,13(13 \%)$ para as diferentes classes. Portanto, devido à contradição que a NBR 7190 (ABNT, 1997) apresenta, são necessários estudos para que possam ser feitas novas avaliações das relações entre as propriedades de resistência à compressão e cisalhamento paralelo às fibras entre diferentes espécies e classes de resistência das madeiras folhosas, visto que as propriedades em questão são de extrema importância para o dimensionamento de estruturas em madeira.

Diante do exposto, o trabalho tem como objetivo investigar, com o auxílio dos ensaios de resistência à compressão paralela às fibras e resistência ao cisalhamento paralelo às fibras, a relação entre essas propriedades; considerando, para tanto, dez espécies de madeira distribuídas nas classes de resistência do grupo das folhosas (madeiras duras). Com os valores característicos das propriedades obtidos experimentalmente, possibilitou-se estimar e avaliar as relações entre as resistências, com o auxílio estatístico do método dos mínimos quadrados, observando se houve divergências nos valores das relações entre as classes de resistências, assim como em relação aos valores estabelecidos pela NBR 7190 (ABNT, 1997).

\section{Material e métodos}

A parte experimental desta pesquisa foi desenvolvida no Laboratório de Madeiras e de Estruturas de Madeira (LaMEM) pertencente ao Departamento de Engenharia de Estruturas (SET), da Escola de Engenharia de São Carlos (EESC), da Universidade de São Paulo (USP). Foram utilizadas peças de madeira serrada de dez espécies: cambará rosa (Erisma sp.), cedro (Cedrella sp.), copaíba (Copaiba resinífera), canafístula (Cassia ferruginea), angelim araroba (Vataireopsis araroba), oiticica amarela (Clarisia racemosa), louro verde (Ocotea sp.), cedroarana (Cedrelinga cateniformis), guaiçara (Luetzelburgia sp.) e maçaranduba (Manikara sp.).

As diferentes espécies de madeira foram adquiridas, no comércio madeireiro local, na forma de tábuas com dimensões próximas de $50 \mathrm{~mm}$ x $90 \mathrm{~mm}$ x $4.000 \mathrm{~mm}$, assim como são obtidas as madeiras utilizadas na construção civil brasileira. Portanto, não foi possível conhecer a origem e idade das árvores.

As tábuas se encontravam em correto armazenamento, ou seja, em pilhas com tabiques apoiadas sobre suportes estáveis espaçados pelo menos $30 \mathrm{~cm}$ do solo no interior do Laboratório de Madeiras e Estruturas em Madeira da EESC - USP.

Os corpos de prova foram confeccionados seguindo as prescrições do Anexo B "Determinação das propriedades das madeiras para projetos de estruturas" da NBR 7190 (ABNT, 1997). Procedeu-se inicialmente à classificação visual das tábuas, então se aferiu o teor de umidade através de um medidor elétrico, modelo M52, da marca Marrari e verificou-se que estavam na umidade padrão de $12 \%$. Em atendimento ao documento normativo brasileiro, adotaram-se corpos de prova isentos de defeitos, retirados aleatoriamente ao longo do lote (para isso, extraiu-se, por ensaio, apenas um corpo de prova por peça) de regiões afastadas das extremidades das tábuas (o menor valor entre cinco vezes a menor dimensão da peça considerada e $30 \mathrm{~cm}$ )

Destaca-se que o documento normativo em questão não faz referência à influência da origem e idade das árvores nas propriedades mecânicas do material; considerando para o dimensionamento estrutural os valores de propriedade correspondentes à respectiva classe de resistência em que se enquadrou a espécie de madeira.

Para cada espécie de madeira foram fabricados 12 corpos de prova para os testes de cisalhamento (Figura 1a), visando à obtenção dos valores de resistência ao cisalhamento na direção paralela às fibras por meio do 
ensaio padronizado (Figura 1b) e outros 12 corpos de prova para os ensaios de compressão (Figura 2a) para a obtenção dos valores de resistência à compressão na direção paralela às fibras por meio do ensaio padronizado (Figura 2b), totalizando 240 amostras fabricadas.

Os ensaios foram realizados na máquina universal de ensaios da marca Amsler (capacidade de carga de 250 $\mathrm{kN}$ ), o carregamento aplicado foi de maneira crescente monotônica à taxa de $10 \mathrm{MPa} / \mathrm{min}$ com dois ciclos de carga e descarga para o ensaio de compressão paralelo às fibras e à taxa de 2,5 MPa/minpara o ensaio de resistência ao cisalhamento paralelo às fibras.

Para o cálculo dos respectivos valores de resistências foram utilizadas as premissas e os métodos de cálculo apresentados no Anexo B da norma brasileira NBR 7190 (ABNT, 1997).

Os valores da resistência ao cisalhamento e à compressão paralela às fibras dos corpos de prova foram obtidos por meio das Equações 1 e 2, respectivamente.

$$
\begin{gathered}
f_{v 0}=\frac{F_{v 0, \text { máx }}}{A_{v}} \\
f_{c 0}=\frac{F_{c 0, \text { máx }}}{A_{c}}
\end{gathered}
$$

Figura 1 - Dimensões em centímetros do corpo de prova (a) para ensaio de resistência ao cisalhamento paralelo às fibras (b) segundo a ABNT NBR 7190 (ABNT, 1997)

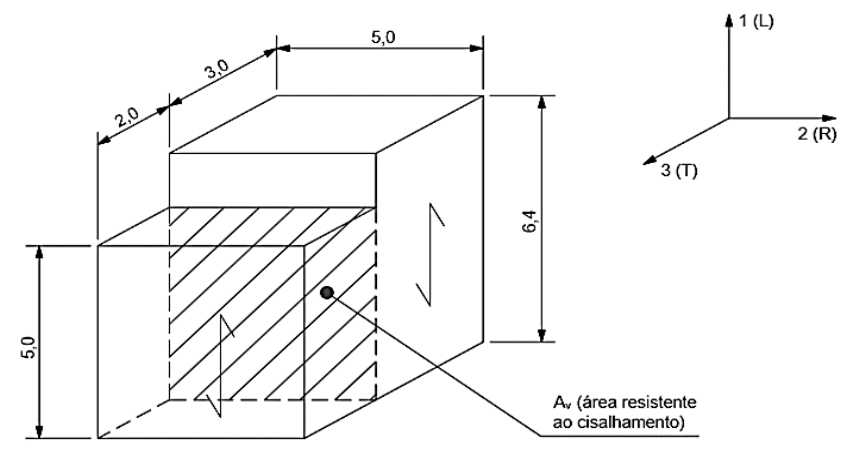

(a)

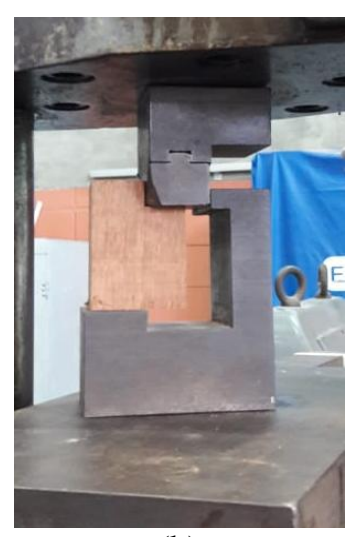

(b)

Figura 2 - Dimensões em centímetros do corpo de prova (a) para ensaio de resistência à compressão paralela às fibras (b) segundo a ABNT NBR 7190:1997

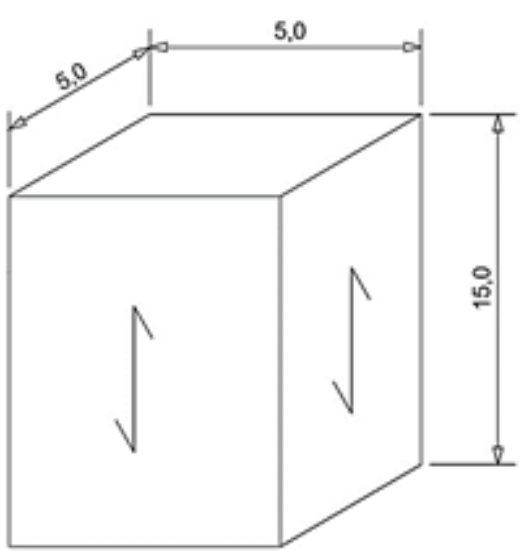

(a)
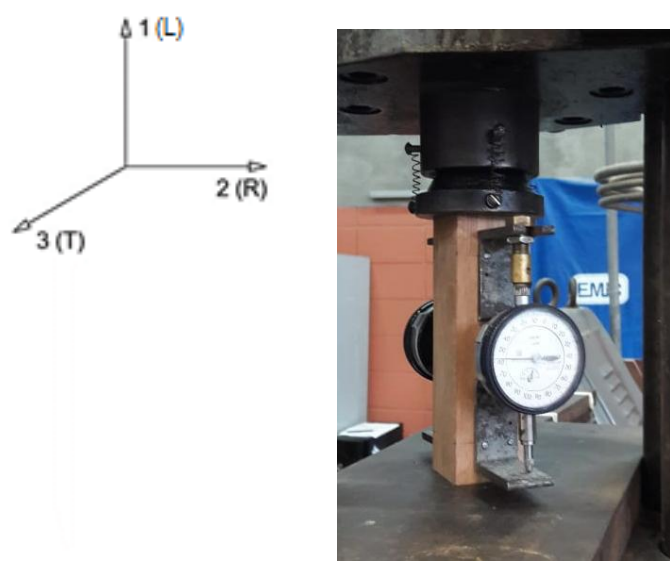

(b)

322 Couto, N. G.; Almeida, J. P. B.; Govone, J. S.; Christoforo, A. L.; Lahr, F. A. R. 
Da Equação 1, $\mathrm{F}_{\mathrm{v} 0 \text {,máx }}$ denota o valor máximo da força cisalhante aplicada no corpo de prova e $\mathrm{A}_{\mathrm{v}}$ é a área inicial da seção crítica do corpo de prova $(5 \mathrm{~cm} \times 5 \mathrm{~cm})$ em um plano paralelo às fibras. Da Equação 2 , $\mathrm{F}_{\mathrm{c} 0 \text {,máx }}$ representa o valor máximo da força de compressão aplicada no corpo de prova e $\mathrm{A}_{\mathrm{c}}$ é a área inicial da seção transversal $(5 \mathrm{~cm} \times 5 \mathrm{~cm})$ do corpo de prova, tomada perpendicularmente à direção de orientação das fibras. Depois de obtidos os valores de resistência pelas experimentações (Equações 1 e 2), os valores de resistência característica $\left(\mathrm{f}_{\mathrm{wk}}\right)$ por espécie de madeira foram calculados com o uso da Equação 3 .

$$
f_{w k}=\left(2 \cdot \frac{f_{1}+f_{2}+f_{3}+\ldots+f_{(n / 2)-1}}{(n / 2)-1}-f_{n / 2}\right) \cdot 1,10
$$

$\mathrm{Na}$ Equação 3 os resultados devem ser colocados em ordem crescente $\left(\mathrm{f}_{1} \leq \mathrm{f}_{2} \leq \mathrm{f}_{3} \ldots \leq \mathrm{f}_{\mathrm{n}}\right)$, desprezando-se o maior valor da resistência em caso de número de corpos de prova ímpar, não se tomando para $\mathrm{f}_{\mathrm{wk}}$ valor de resistência inferior a $f_{1}$ e nem inferior a 0,70 do valor médio da resistência.

Com posse dos valores característicos obtidos da Equação 3 para cada espécie de madeira (10 valores de $\mathrm{f}_{\mathrm{v} 0, \mathrm{k}}$ e 10 valores de $\mathrm{f}_{\mathrm{c} 0, \mathrm{k}}$ ), foi realizada a análise estatística com o auxílio do software Mathcad ${ }^{\circledR}$ versão 14 . Obteve-se o coeficiente ótimo $(\lambda)$ da relação entre os valores de resistência $\left(f_{v 0, k}=\lambda \cdot f_{c 0, k}\right)$ através da minimização da Equação 4 (mínimos quadrados) com o auxílio do método de Newton com aproximação quadrática (Equação 5), ficando a convergência para solução ótima independente da escolha da estimativa inicial $\lambda_{\mathrm{o}}$ do método iterativo.

$$
f(\lambda)=\frac{1}{2} \sum_{i=1}^{n}\left(f_{v 0, k_{i}}-\lambda \cdot f_{c 0, k_{i}}\right)^{2}
$$

O coeficiente $\lambda$ obtido da Equação 5 foi comparado com o coeficiente proposto pela norma brasileira, objetivando-se verificar a diferença entre ambos.

$$
\lambda=\lambda_{o}-\left(\frac{d^{2}}{d \lambda^{2}}\left[f\left(\lambda_{o}\right)\right]\right)^{-1} \cdot \frac{d}{d \lambda}\left[f\left(\lambda_{o}\right)\right]
$$

\section{Resultados e discussão}

A Tabela 1 apresenta, para cada uma das espécies, os valores médios de resistência calculados pelas

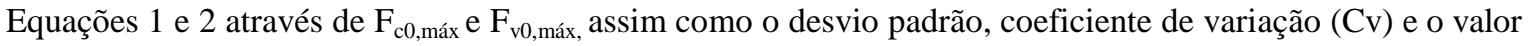

\begin{tabular}{|c|c|c|c|c|c|c|c|c|c|c|}
\hline \multirow[t]{2}{*}{ Estatística } & \multicolumn{2}{|c|}{$\begin{array}{c}\text { Cambará } \\
\text { rosa }\end{array}$} & \multicolumn{2}{|c|}{ Cedro } & \multicolumn{2}{|c|}{ Copaíba } & \multicolumn{2}{|c|}{ Canafístula } & \multicolumn{2}{|c|}{$\begin{array}{c}\text { Angelim } \\
\text { araroba }\end{array}$} \\
\hline & $\mathbf{f}_{\mathrm{c} 0}$ & $\mathbf{f}_{\mathrm{v} 0}$ & $\mathbf{f}_{\mathrm{c} 0}$ & $\mathbf{f}_{\mathrm{v} 0}$ & $\mathbf{f}_{\mathrm{c} 0}$ & $\mathbf{f}_{\mathrm{v} 0}$ & $\mathbf{f}_{\mathrm{c} 0}$ & $\mathbf{f}_{\mathrm{v} 0}$ & $\mathbf{f}_{\mathrm{c} 0}$ & $\mathbf{f}_{\mathrm{v} 0}$ \\
\hline Desvio padrão & 4,98 & 1,18 & 5,51 & 2,38 & 5,83 & 2,85 & 9,81 & 5,19 & 6,27 & 4,15 \\
\hline Média & 33,8 & 7,3 & 30,8 & 9,1 & 49,1 & 14,2 & 51,2 & 18,5 & 50,7 & 9,8 \\
\hline Coef. de variação & 0,15 & 0,16 & 0,18 & 0,26 & 0,12 & 0,20 & 0,19 & 0,28 & 0,12 & 0,42 \\
\hline \multirow[t]{3}{*}{ Valor característico } & 25,67 & 5,95 & 29,36 & 6,98 & 43,53 & 10,62 & 31,83 & 9,20 & 44,24 & 4,38 \\
\hline & \multicolumn{2}{|c|}{$\begin{array}{c}\text { Oiticica } \\
\text { amarela }\end{array}$} & \multicolumn{2}{|c|}{ Guaiçara } & \multicolumn{2}{|c|}{ Louro verde } & \multicolumn{2}{|c|}{ Cedroarana } & \multicolumn{2}{|c|}{ Maçaranduba } \\
\hline & $\mathbf{f}_{\mathrm{c} 0}$ & $\mathbf{f}_{\mathrm{v} 0}$ & $\mathbf{f}_{\mathrm{c} 0}$ & $\mathbf{f}_{\mathrm{v} 0}$ & $\mathbf{f}_{\mathrm{c} 0}$ & $\mathbf{f}_{\mathrm{v} 0}$ & $\mathbf{f}_{\mathrm{c} 0}$ & $\mathbf{f}_{\mathrm{v} 0}$ & $\mathbf{f}_{\mathrm{c} 0}$ & $\mathbf{f}_{\mathrm{v} 0}$ \\
\hline Desvio padrão & 6,86 & 2,10 & 8,89 & 2,17 & 5,67 & 1,03 & 9,46 & 3,13 & 6,18 & 2,78 \\
\hline Média & 70,3 & 17,8 & 71,1 & 20,7 & 52,9 & 11,0 & 41,5 & 12,0 & 82,9 & 24,9 \\
\hline Coef. de variação & 0,10 & 0,12 & 0,12 & 0,10 & 0,11 & 0,09 & 0,23 & 0,26 & 0,07 & 0,11 \\
\hline Valor característico & 62,41 & 15,18 & 60,10 & 17,51 & 49,14 & 9,77 & 24,84 & 5,90 & 79,46 & 20,77 \\
\hline
\end{tabular}
característico calculado através da Equação 3.

Tabela 1 - Resultados obtidos experimentalmente 
Com exceção da madeira de angelim araroba, que obteve, para a resistência ao cisalhamento paralelo às fibras $\left(\mathrm{f}_{\mathrm{v} 0}\right), \mathrm{Cv}$ correspondente a $42 \%$, as espécies apresentaram valores de $\mathrm{Cv}$ dentro do estabelecido pela norma brasileira NBR 7190 (ABNT, 1997), sendo 18\% o máximo valor para solicitações normais e 28\% para solicitações tangenciais. Ressalta-se que dispersões nos resultados podem ser justificadas, em partes, pela variabilidade intrínseca do material e pelo modelo de cálculo utilizado para a determinação dos resultados experimentais.

Como pode ser observado pelos valores característicos da resistência à compressão constantes na Tabela 1, as espécies ensaiadas apresentaram classificação diversa quanto às classes de resistências (proposta pela NBR 7190 (ABNT, 1997) na Tabela 9 do item 6.3.5), sendo um total de três espécies classificadas como C20 (cedro, cambará rosa, cedroarana), uma como C30 (canafístula), três como C40 (louro verde, angelim araroba e copaíba) e três espécies pertencentes à classe C60 (oiticica amarela, guaiçara e maçaranduba).

Para a madeira de cambará rosa (Erisma sp.) obteve-se valor médio de resistência à compressão paralela às fibras $\left(\mathrm{f}_{\mathrm{c} 0}\right)$ de $33,8 \mathrm{MPa}$, sendo inferior ao apresentado pela NBR 7190 (ABNT, 1997) [37,8 MPa quarubarana (Erisma uncinatum)] e por Arruda, Menezzi e Andrade (2015) (49,75 MPa). Quanto à resistência ao cisalhamento paralelo às fibras $\left(\mathrm{f}_{\mathrm{v} 0}\right)$, o valor de 7,98 MPa encontrado foi divergente do de 14 MPa encontrado por Lahr et al. (2016) e próximo ao apresentado pela norma brasileira NBR 7190 (ABNT, 1997) [5,8 Mpa - quarubarana (Erisma uncinatum)].

Em relação às propriedades do cedro (Cedrella sp.), a resistência ao cisalhamento paralelo às fibras apresentou valor médio de 9,1 MPa, sendo superior ao apresentado pela NBR 7190 (ABNT, 1997) [5,6 MPa - cedro doce (Cedrella spp.)]. O valor médio de resistência à compressão paralela às fibras obtido foi de $30,8 \mathrm{MPa}$, sendo próximo ao que a NBR 7190 (ABNT, 1997) apresenta [(31,5 MPa - cedro doce (Cedrella spp.)] e do encontrado por Dias e Lahr (2004) (33 MPa).

Para a madeira de canafístula (Cassia ferruginea), a resistência à compressão paralela às fibras apresentou valor médio de 51,2 MPa, que foi maior que o obtido por Vivian et al. (2010) (30,09 MPa) e próximo ao valor apresentado pela NBR 7190 (ABNT, 1997) (52 MPa) por Dias e Lahr (2004) (54 MPa) e Almeida et al. (2016) (51,96 MPa). Em relação ao $\mathrm{f}_{\mathrm{v} 0}$ encontrado (18,5 MPa) foi superior aos apresentados por Vivian et al. (2010) (13,04 MPa) e pela NBR 7190 (ABNT, 1997) (11,1 MPa).

A madeira de angelim araroba (Vataireopsis aroroba), atingiu 50,7 MPa de resistência à compressão paralela às fibras, sendo esse valor equivalente ao apresentado por Almeida et al. (2016) (50,93 MPa), Grobério e Lahr (2002) (50,2 MPa) e pela NBR 7190 (ABNT, 1997) (50,5 MPa). Para a resistência ao cisalhamento paralelo às fibras, o valor médio encontrado foi de $9,8 \mathrm{MPa}$, sendo superior ao apresentado pela NBR 7190 (ABNT, 1997) (7,1 MPa) e inferior aos obtidos por Grobério e Lahr (2002) (11,3 MPa) e Dias e Lahr (2004) (12 MPa).

Em relação às propriedades da oiticica amarela (Clarisia racemosa), a $\mathrm{f}_{\mathrm{c} 0}$ foi de70,3 $\mathrm{MPa}$, apresentando resultados próximos ao apresentado pela NBR 7190 (ABNT, 1997) (69,9 MPa). Para o $\mathrm{f}_{\mathrm{v} 0}$ o valor médio obtido (17,8 MPa) foi superior ao apresentado pela norma brasileira (10,6 MPa).

Já para a maçaranduba (Manilkara sp.), a resistência à compressão paralela às fibras foi de 82,9 MPa, apresentando valor superior ao obtido por Jesus, Logsdon e Finger (2015) (67,57 MPa) e idêntico ao da NBR 7190 (ABNT, 1997) (82,9 MPa). Para a resistência ao cisalhamento paralelo às fibras, o valor médio encontrado foi de 24,9 MPa sendo superior aos apresentados pela norma brasileira (14,9 MPa) e Jesus, Logsdon e Finger (2015) (11,77 MPa).

Para a madeira de cedroarana (Cedrelinga cateniformis), os valores apresentados por Jesus, Logsdon e Finger (2015) (30,53 MPa), Moreira et al. (2017) (31 MPa) e Dias e Lahr (2004) (33 MPa) foram inferiores aos obtidos no presente estudo $(41,3 \mathrm{MPa})$ para a resistência à compressão paralela às fibras. Para a resistência ao cisalhamento paralelo às fibras, que apresentou o valor médio de $12 \mathrm{MPa}$, os autores Jesus, Logsdon e Finger (2015) (6,21 MPa) e Dias e Lahr (2004) (9,4 MPa) também obtiveram valores inferiores.

A resistência à compressão paralela às fibras da madeira de louro verde (Ocotea sp.) resultou em um valor médio de 52,9 MPa, sendo esse inferior ao determinado por Araújo (2007) (61,98 MPa) e próximo aos apresentados pela NBR 7190 (ABNT, 1997) [56,5 MPa - louro preto (Ocotea spp.)] e por Dias e Lahr (2004) (55 MPa). Em relação à resistência ao cisalhamento paralelo às fibras, o presente estudo resultou em um valor médio de $11 \mathrm{MPa}$, sendo próximo aos encontrados por Araújo (2007) (9,71 MPa), Dias e Lahr (2004) (13,1 MPa) e NBR 7190 (ABNT, 1997) [9 MPa - louro preto (Ocotea spp)].

Para a madeira de guaiçara (Luetzelburgia sp.), a resistência à compressão paralela às fibras apresentou valor médio de 71,1 Mpa, sendo superior ao encontrado por Dias e Lahr (2004) (66 MPa) e muito próximo ao

324 Couto, N. G.; Almeida, J. P. B.; Govone, J. S.; Christoforo, A. L.; Lahr, F. A. R. 
apresentado no Anexo E da norma brasileira NBR 7190 (ABNT, 1997) (71,4 MPa). Em relação à resistência ao cisalhamento paralelo às fibras, o valor médio encontrado no presente estudo foi de 20,7 MPa, sendo superior ao apresentado pela norma brasileira NBR 7190 (ABNT, 1997) (12,5 MPa) e por Dias e Lahr (2004) (19,0 MPa).

As Tabelas 2 e 3 apresentam os resultados para as relações encontradas entre as propriedades considerando cada espécie individualmente e separadas por classes de resistência, respectivamente. O coeficiente ótimo ( $\lambda$ ) para as relações apresentadas foram obtidos através da minimização da Equação 4.

Por fim, calculou-se a relação considerando todas as espécies no mesmo conjunto. O coeficiente ótimo $(\lambda)$, aplicando a estatística apresentada nas Equações 4 e 5, é apresentada na Equação 6.

$f_{v 0, k}=0,221 . f_{c 0, k}$

Eq. 6

Como pode ser observado na Equação 6, constata-se em uma relação que a resistência ao cisalhamento paralelo às fibras é da ordem de $22,1 \%$ da resistência à compressão paralela às fibras, sendo $84,16 \%$ maior do que o valor proposto pela NBR 7190 (ABNT, 1997) (12\%). A Tabela 3 evidencia que não há variação quando o valor do coeficiente ótimo é reduzido conforme a classe de resistência sofre acréscimo, conforme o texto da NBR 7190 (ABNT, 1997) determina na Tabela 9 (página 16). A divergência apresentada entre o valor proposto pela norma e a Equação 6 pode ter como um dos fundamentos o fato de que a relação apresentada no item 6.3.3 da NBR 7190 (ABNT, 1997) considera as madeiras de reflorestamento e nativas no mesmo conjunto, sendo que as madeiras de reflorestamento tendem a apresentar valores de resistências inferiores às nativas, e assim influenciando no coeficiente ótimo $(\lambda)$ da relação.

Matos e Molina (2016) encontraram para madeira de Eucalyptus saligna uma madeira folhosa de reflorestamento, o coeficiente de 0,13 para a relação $\mathrm{f}_{\mathrm{v} 0, \mathrm{k}} / \mathrm{f}_{\mathrm{c} 0, \mathrm{k}}$. Nota-se que o valor encontrado foi inferior ao apresentado pelo presente estudo e próximo ao apresentado pela NBR 7190 (ABNT, 1997), reforçando a possível influência das madeiras reflorestadas na relação investigada.

Tabela 2 - Relações entre as propriedades para cada espécie

\begin{tabular}{l|c}
\hline \multicolumn{1}{c|}{ Espécie } & \multicolumn{1}{c}{ Relação } \\
\hline Angelim araroba & $\mathrm{f}_{\mathrm{v} 0, \mathrm{k}}=0,10 \cdot \mathrm{f}_{\mathrm{c} 0, \mathrm{k}}$ \\
Cambará rosa & $\mathrm{f}_{\mathrm{v} 0, \mathrm{k}}=0,23 \cdot \mathrm{f}_{\mathrm{c} 0, \mathrm{k}}$ \\
Canafístula & $\mathrm{f}_{\mathrm{v} 0, \mathrm{k}}=0,29 \cdot \mathrm{f}_{\mathrm{c} 0, \mathrm{k}}$ \\
Cedro & $\mathrm{f}_{\mathrm{v} 0, \mathrm{k}}=0,24 \cdot \mathrm{f}_{\mathrm{c} 0, \mathrm{k}}$ \\
Cedroarana & $\mathrm{f}_{\mathrm{v} 0, \mathrm{k}}=0,24 \cdot \mathrm{f}_{\mathrm{c} 0, \mathrm{k}}$ \\
Copaíba & $\mathrm{f}_{\mathrm{v} 0, \mathrm{k}}=0,24 \cdot \mathrm{f}_{\mathrm{c} 0, \mathrm{k}}$ \\
Guaiçara & $\mathrm{f}_{\mathrm{v} 0, \mathrm{k}}=0,19 \cdot \mathrm{f}_{\mathrm{c} 0, \mathrm{k}}$ \\
Louro verde & $\mathrm{f}_{\mathrm{v} 0, \mathrm{k}}=0,19 \cdot \mathrm{f}_{\mathrm{c} 0, \mathrm{k}}$ \\
Maçaranduba & $\mathrm{f}_{\mathrm{v} 0, \mathrm{k}}=0,26 \cdot \mathrm{f}_{\mathrm{c} 0, \mathrm{k}}$ \\
Oiticica amarela & $\mathrm{f}_{\mathrm{v} 0, \mathrm{k}}=0,24 \cdot \mathrm{f}_{\mathrm{c} 0, \mathrm{k}}$ \\
\hline \multicolumn{1}{c|}{ Média } & $\mathbf{f}_{\mathrm{v} 0, \mathrm{k}}=\mathbf{0 , 2 2} \cdot \mathbf{f}_{\mathrm{c} 0, \mathbf{k}}$ \\
\hline \multicolumn{1}{c}{ Desv. padrão } & $\mathbf{0 , 0 5 1}$ \\
\hline \multicolumn{1}{c|}{ Coef. variação } & $\mathbf{0 , 2 3}$ \\
\hline
\end{tabular}

Tabela 3 - Relações entre as propriedades para cada classe de resistência

\begin{tabular}{c|c|c}
\hline Classe & $\begin{array}{c}\text { Coeficiente obtido no } \\
\text { presente estudo }\end{array}$ & NBR 7190 (ABNT,1997) \\
\hline C20 & $\mathrm{f}_{\mathrm{v} 0, \mathrm{k}}=0,24 \cdot \mathrm{f}_{\mathrm{c} 0, \mathrm{k}}$ & $\mathrm{f}_{\mathrm{v} 0, \mathrm{k}}=0,20 \cdot \mathrm{f}_{\mathrm{c} 0, \mathrm{k}}$ \\
$\mathrm{C} 30$ & $\mathrm{f}_{\mathrm{v} 0, \mathrm{k}}=0,29 \cdot \mathrm{f}_{\mathrm{c} 0, \mathrm{k}}$ & $\mathrm{f}_{\mathrm{v} 0, \mathrm{k}}=0,16 \cdot \mathrm{f}_{\mathrm{c} 0, \mathrm{k}}$ \\
$\mathrm{C} 40$ & $\mathrm{f}_{\mathrm{v} 0, \mathrm{k}}=0,18 \cdot \mathrm{f}_{\mathrm{c} 0, \mathrm{k}}$ & $\mathrm{f}_{\mathrm{v} 0, \mathrm{k}}=0,15 \cdot \mathrm{f}_{\mathrm{c} 0, \mathrm{k}}$ \\
C60 & $\mathrm{f}_{\mathrm{v} 0, \mathrm{k}}=0,23 \cdot \mathrm{f}_{\mathrm{c} 0, \mathrm{k}}$ & $\mathrm{f}_{\mathrm{v} 0, \mathrm{k}}=0,13 \cdot \mathrm{f}_{\mathrm{c} 0, \mathrm{k}}$ \\
\hline Média & $\mathbf{f}_{\mathbf{v} \mathbf{0}, \mathbf{k}}=\mathbf{0 , 2 3} \cdot \mathbf{f}_{\mathbf{c} \mathbf{0}, \mathbf{k}}$ & $\mathbf{f}_{\mathrm{v} \mathbf{0}, \mathbf{k}}=\mathbf{0 , 1 6} \cdot \mathbf{f}_{\mathbf{c} 0, \mathbf{k}}$ \\
\hline
\end{tabular}




\section{Conclusão}

$\mathrm{O}$ valor encontrado para a relação entre a resistência ao cisalhamento paralela às fibras e a resistência à compressão paralela às fibras no presente estudo considerando o conjunto de todas as espécies foi $84,16 \%$ superior ao valor proposto pela NBR 7190 (ABNT, 1997). Destaca-se que ao apresentar uma relação com valor inferior, é possível afirmar que a norma brasileira está conservadora. Essa divergência faz com que haja superdimensionamento dos elementos, e consequentemente maior gasto de material e aumento do peso próprio das estruturas.

Os resultados considerando as diferentes classes de resistência também apresentaram divergências com os valores da norma brasileira, sendo que os resultados do presente estudo não apresentaram decréscimos nos valores dos coeficientes ótimos das relações à medida que as classes de resistência vão aumentando. Dessa forma, sugere-se que os lotes de madeira estejam acompanhados de laudo de análise de resistência mecânica, evitando o uso inadequado da madeira. Um dos possíveis fundamentos para as divergências encontradas entre os resultados do presente estudo e dos valores apresentados pela NBR 7190 (ABNT, 1997) é o fato de que a relação apresentada no item 6.3.3 da NBR 7190 (ABNT, 1997) considera as madeiras de reflorestamento e nativas no mesmo conjunto.

Em função da grande diferença encontrada entre os valores das relações em comparação com os estabelecidos pela norma, faz-se necessário o desenvolvimento de outras pesquisas nessa temática, de maneira a se aumentar o número de espécies de madeira e consequentemente a confiabilidade associada à determinação do coeficiente da relação aqui investigada. Ressalta-se também a importância da investigação considerando madeiras de reflorestamento e nativas de maneira separada, além de outras relações entre propriedades apresentadas na norma brasileira NBR 7190 (ABNT, 1997), e assim haver contribuição para uma possível revisão da norma.

\section{Referências}

ALMEIDA, D. H. et al. Tenacidade da madeira como função da densidade aparente. Revista Árvore, Viçosa, v. 38, n. 1, p. 203-207, 2014.

ALMEIDA, T. H. et al. Density as estimator of strength in compression parallel to the grain in wood. International Journal of Materials Engineering, v. 6, n. 3, p. 67-71, 2016.

ARAUJO, H. J. B. Relações funcionais entre propriedades físicas e mecânicas de madeiras tropicais brasileiras. Floresta, Curitiba, v. 37, n. 3, set./dez. 2007.

ARRUDA, L. M.; MENEZZI, C. H. S. D.; ANDRADE, A. Utilization of a thermomechanical process to enhance properties of hardwood used for flooring. Brazilian Journal of Wood Science, v. 6, n. 3, p. 223$231,2015$.

ASSOCIAÇÃO BRASILEIRA DE NORMAS TÉCNICAS. NBR 7190: projeto de estruturas de madeira.Rio de Janeiro, 1997.

BEECH, E. et al. GlobalTreeSearch: the first complete global database of tree species and country distributions. Journal of Sustainable Forestry, v. 36, n. 5, p. 454-489, 2017.

CALIL JÚNIOR, C.; DIAS, A. A. Utilização da madeira em construções rurais. Revista da Madeira, v. 88, 2005.

CHRISTOFORO, A. L. et al. Characterization of tropical wood species for use in civil constructions. Journal of Civil Engineering Research, v. 3, n. 3, p. 98-103, 2013.

CURY, G.; TOMAZELLO FILHO, M. Descrição anatômica de espécies de madeira utilizadas na Construção Civil. Floresta e Ambiente, v. 18, n. 3, p. 227-236, 2011.

DIAS, F. M.; LAHR, F. A. R. Estimativa de propriedades de resistência e rigidez da madeira através da densidade aparente. Scientia Florestalis, Piracicaba, v. 65, p. 102-113, jun. 2004.

GROBÉRIO, M. P.; LAHR, F. A. R. Indicações para o emprego da madeira de espécies tropicais do Brasil. Madeira: Arquitetura e Engenharia, v. 8, 2002.

HEMSTROM, K. M. L. G. Multi-storey wood-frame buildings in Germany, Sweden and the UK. Construction Innovation, v. 12, n. 1, p. 62-85, 2016. 
INDÚSTRIA BRASILEIRA DE ÁRVORES. Relatório Anual. 2017. Disponível em: https://iba.org/images/shared/Biblioteca/IBA_RelatorioAnual2017.pdf. Acesso em: 10 jun. 2018.

JESUS, J. M. H.; LOGSDON, N. B.; FINGER, Z. Classes de resistência de algumas madeiras de Mato Grosso. Engineering and Science, v. 1, n. 3, 2015.

LAHR, F. A. R. et al. Full Characterization of Erisma uncinatum Warm Wood Specie. International Journal of Materials Egineering, v. 6, n. 5, p. 147-150, 2016.

LAROCA, C.; MATOS, J. Habitação social e design como oportunidades para o setor. Revista da madeira, n. 77, 2003.

MATOS, G. S.; MOLINA, J. C. Resistência da madeira ao cisalhamento paralelo às fibras segundo as normas ABNT NBR 7190:1997 e ISSO 13910:2005. Revista Matéria, v. 21, n. 4, p. 1069-1079, 2016.

MORAES, P. D.; FIGUEROA, M. J. M. Comportamento da madeira a temperaturas elevadas. Ambiente Construído, Porto Alegre, v. 9, n. 4, p. 157-174, out./dez. 2009.

MOREIRA, A. P. et al. Toughness and impact strength in dynamic bending of wood as a function of the modulus of elasticity and the strength in compression to the grain. International Journal of Materials Engineering, v. 7, n. 4, p. 61-67, 2017.

REMAGE, M. H. et al. The wood from the trees: the use of timber in construction. Renewable and Sustainable Energy, v. 68, p. 333-359, 2017.

SISTEMA FLORESTAL BRASILEIRO. Recursos florestais. Boletim SNIF 2016, v. 7, n. 2, p.7, 2016.

VIVIAN, M. A. et al. Propriedades físico-mecânicas da madeira de Canafístula aos 10 anos de idade.

Ciência Rural, Santa Maria, v. 40, n. 5, p. 1097-1102, 2010.

WANG, L.; TOPPINEN, A.; JUSLIN, H. Use of wood in green building: a study of expert perspectives from the UK. Journal of Cleaner Production, v. 65, p. 350-361, 2014.

Natalia Garcia Couto

Departamento de Engenharia Civil | Universidade Federal de São Carlos | Rodovia Washington Luís, km 235, s/n, Jardim Guanabara | São Carlos - SP - Brasil | CEP 13565-905 | Tel.: (16) 3351-8262 | E-mail: natygcouto@gmail.com

\section{João Paulo Boff Almeida}

Departamento de Engenharia Civil | Universidade Federal de São Carlos | E-mail: boff.joaopaulo@gmail.com

José Silvio Govone

Departamento de Estatística, Matemática Aplicada e Computação | Universidade Estadual Paulista Julio de Mesquita Filho | Av. 24 A, 1505, Bela Vista | Rio Claro - SP - Brasil | CEP 13506-970 | Tel.: (19) 3526-9080 | E-mail: jsgovone@gmail.com

\section{André Luis Christoforo}

Departamento de Engenharia Civil | Universidade Federal de São Carlos | E-mail: mchristoforoal@yahoo.com.br

\section{Francisco Antonio Rocco Lahr}

Departamento de Engenharia Civil | Universidade de São Paulo | Av. Trabalhador São Carlense, 400, Centro | São Carlos - SP - Brasil | CEP 13566-590 | Tel.: (16) 3373-9483 | E-mail: frocco@sc.usp.br

\section{Ambiente Construído}

Revista da Associação Nacional de Tecnologia do Ambiente Construído

Av. Osvaldo Aranha, $99-3^{\circ}$ andar, Centro

Porto Alegre - RS - Brasil CEP 90035-190

Telefone: +55 (51) 3308-4084

Fax: +55 (51) 3308-4054

www.seer.ufrgs.br/ambienteconstruido

E-mail: ambienteconstruido@ufrgs.br 